Revue d'histoire de l'enfance « irrégulière »

Le Temps de l'histoire

$21 \mid 2019$

L'étrange étrangeté des dossiers de l'enfance irrégulière

\title{
Les fonds des établissements du secteur public de la PJJ conservés aux archives départementales du Val- de-Marne
}

Agnès Seguin et Zoï Kyritsopoulos

\section{(2) OpenEdition}

Journals

Édition électronique

URL : https://journals.openedition.org/rhei/4885

DOI : 10.4000/rhei.4885

ISSN : $1777-540 X$

Éditeur

Presses universitaires de Rennes

Édition imprimée

Date de publication : 12 novembre 2019

Pagination : 209-218

ISBN : 978-2-7535-7844-9

ISSN : $1287-2431$

Référence électronique

Agnès Seguin et Zoï Kyritsopoulos, «Les fonds des établissements du secteur public de la PJJ

conservés aux archives départementales du Val-de-Marne », Revue d'histoire de l'enfance « irrégulière » [En ligne], 21 | 2019, mis en ligne le 12 novembre 2021, consulté le 05 décembre 2022. URL : http:// journals.openedition.org/rhei/4885; DOI : https://doi.org/10.4000/rhei.4885

Ce document a été généré automatiquement le 5 décembre 2022

Tous droits réservés 


\title{
Les fonds des établissements du secteur public de la PJJ conservés aux archives départementales du Val-de-Marne
}

\author{
Agnès Seguin et Zoï Kyritsopoulos
}

1 Les archives départementales du Val-de-Marne se sont enrichies à l'été 2017 du fonds du centre d'orientation et de rééducation de Chevilly-Larue qui représente quasiment $130 \mathrm{ml}$ de documents couvrant les années 1930 à 2015 (et dont quelques rares pièces remontent au XIX ${ }^{\mathrm{e}}$ siècle).

2 Cet ensemble massif est très composite : il correspond en fait à des archives produites par différents établissements d'accueil de filles mineures ayant fonctionné sur le site de Chevilly-Larue. En 1907, la communauté Notre-Dame de la Charité est la première à s'y implanter, en transférant son refuge auparavant basé sur Paris. En 1930, la communauté reçoit l'agrément public lui permettant d'accueillir des mineures placées par le tribunal pour enfants, pour lesquelles différents modes d'accueil et de prise en charge seront successivement développés. En 1977, l'ensemble de la structure est repris par l'association Saint-Michel-des-Sorbiers, avant la fondation Rothschild en 2010.

3 L'ensemble versé en 2017 regroupe des archives issues de l'activité de ces différents producteurs et comprend au final :

4 - 6 articles concernant l'administration générale, surtout intéressants grâce aux rapports d'activité de 1961 à 2002 ;

$5-3$ articles relatifs à l'organisation et aux activités des différents établissements entre les années 1930 et 2015 ;

6 - les autres articles, qui correspondent à la très grande majorité du fonds, portent sur la prise en charge des pensionnaires, avec 43 registres de présence et/ou d'entrée, des fiches mécanographiques (13 boîtes), et surtout une collection massive de dossiers individuels (1444 boîtes concernant les pensionnaires entrées de 1938 à 2014). 
7 Parmi cette dernière typologie, les dossiers les plus riches et originaux sont ceux du centre expérimental d'observation, créé en 1948 par le docteur Paul Le Moal, neuropsychiatre, et notamment exploités par Véronique Blanchard dans le cadre de sa thèse "Mauvaises filles»: portraits de la déviance féminine juvénile (1945-1958). C'est d'ailleurs Véronique Blanchard et Mathias Gardet qui prennent contact en 2016 avec les archives départementales du Val-de-Marne afin d'organiser en priorité la sauvegarde de cette partie du fonds, menacé suite à la restructuration de l'établissement engagée par la fondation Rothschild.

\section{Illustration 1}

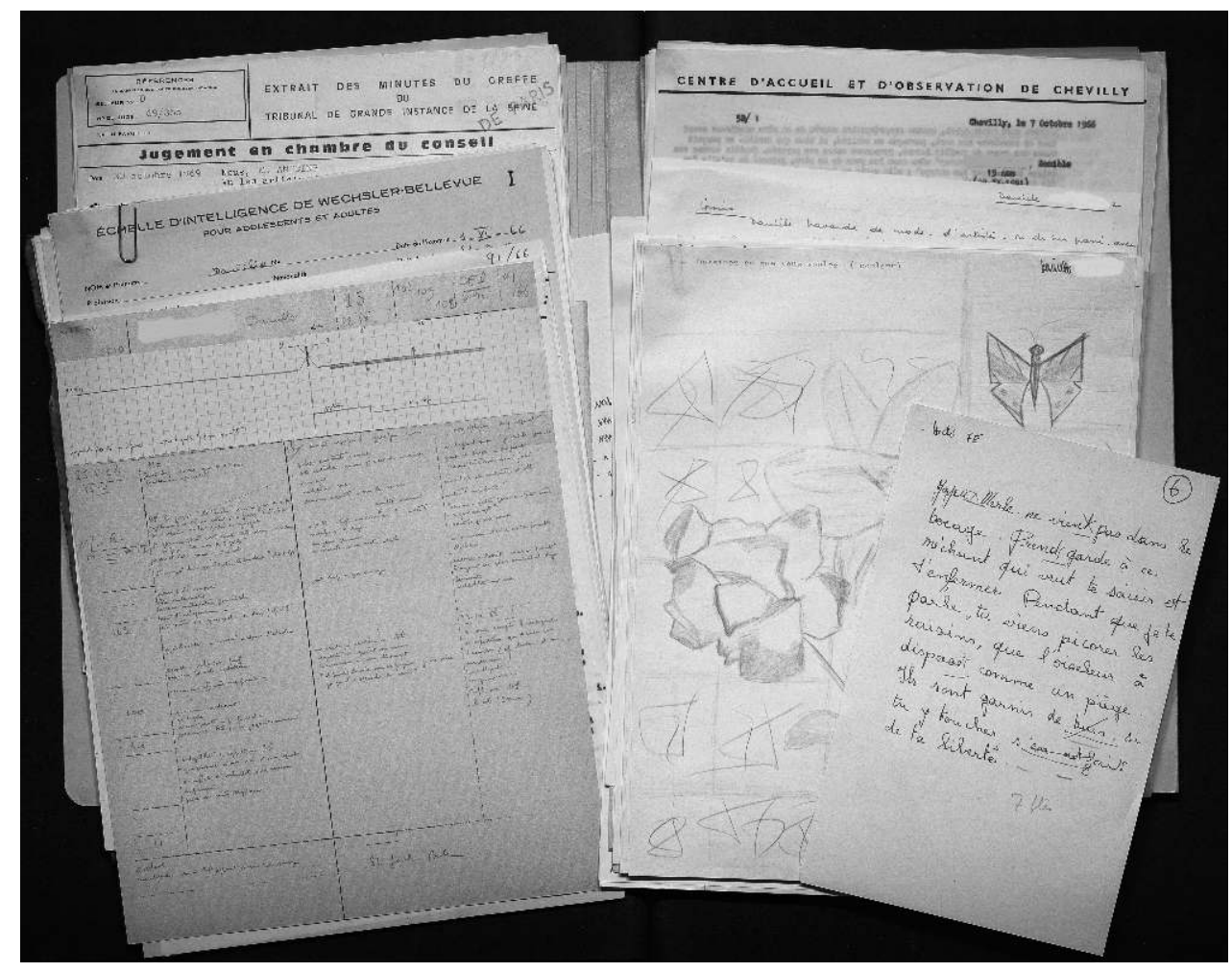

Les archives départementales du Val-de-Marne choisissent de ne pas se limiter à la période du centre d'observation et d'effectuer une collecte plus large, allant jusqu'au milieu des années 2010. En effet, même si les dossiers postérieurs aux années 1970 ne présentent plus la même richesse documentaire, le fonds est très bien tenu depuis la fin des années 1930 et a été très peu mouvementé, l'établissement possédant des espaces de stockage suffisants. Ainsi, conserver l'ensemble permet de mettre en valeur les évolutions des formes d'accueil et de prise en charge éducative des jeunes filles dans le département sur une période longue. 
Illustration 2

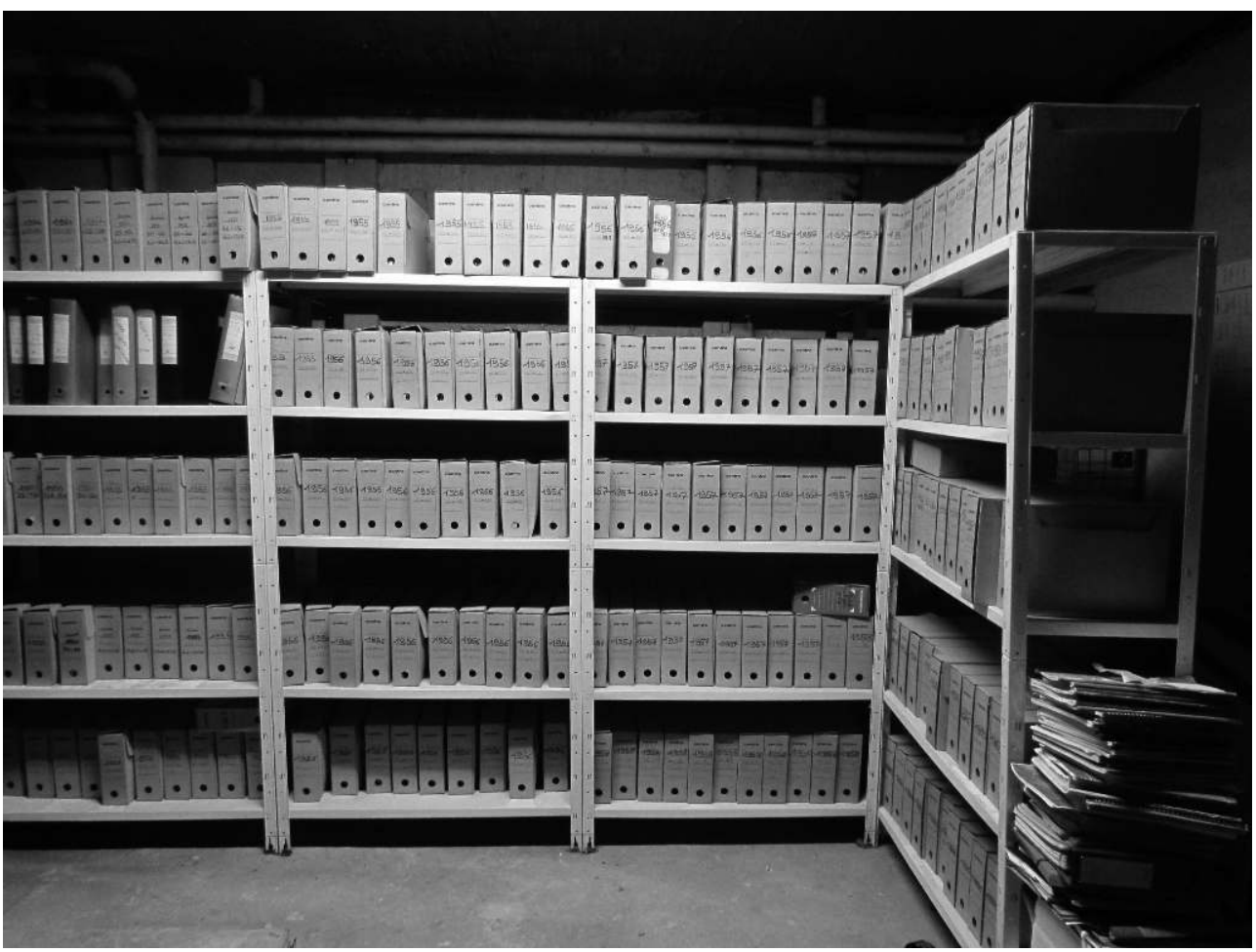

Illustration 3

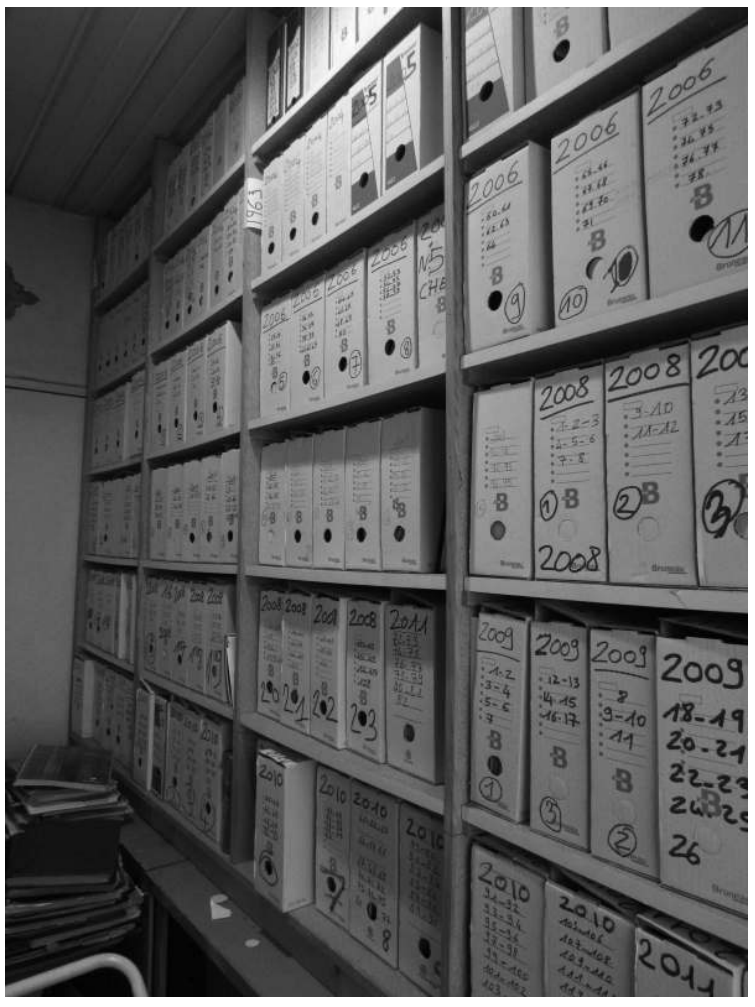

9 Face à la richesse de ce fonds, à la masse, et à la nécessité d'opérer dans l'urgence, les archives départementales prennent elles-mêmes en charge le déménagement et la rédaction d'un premier bordereau de versement au cours de l'été 2017. L'objectif est 
alors de pouvoir mettre rapidement ce fonds à disposition afin de répondre aux sollicitations des chercheurs. Au final, le travail conjoint avec Mathias Gardet et Véronique Blanchard a permis que ces archives soient rapidement exploitées pour des travaux de recherche ou des ateliers avec de futurs éducateurs.

Ces archives rejoignent les autres fonds déjà conservés par les archives départementales du Val-de-Marne sur cette thématique. Même si aucune campagne systématique de collecte n'a été organisée, les structures relevant de la Protection judiciaire de la jeunesse (PJJ) dans le département ont eu le réflexe, dès la fin des années 1980, de saisir les archives en cas de problème de place ou lors d'un déménagement. $\mathrm{Au}$ final, toutes n'ont pas versé, mais l'ensemble actuellement constitué couvre quasiment toutes les périodes depuis le début du $\mathrm{xx}^{\mathrm{e}}$ siècle.

Tableau des versements des structures relevant de la Protection judiciaire de la jeunesse par ordre alphabétique des communes d'implantation

\begin{tabular}{|c|c|c|c|c|}
\hline Commune & Type d'établissement & & Versement & $\begin{array}{l}\text { Dates des } \\
\text { documents }\end{array}$ \\
\hline Arcueil & $\begin{array}{l}\text { Centre d'orientation et d'action } \\
\text { éducative (COAE) }\end{array}$ & $1809 \mathrm{~W} 1-212$ & $\begin{array}{l}\text { Fonctionnement du centre : rapports, registres, dossiers. } \\
\text { Consultations: dossiers individuels. }\end{array}$ & 1958-1992 \\
\hline Charenton-le-Pont & Centre d'éducation surveillée (CES) & $\begin{array}{c}1547 \mathrm{~W} 1 \\
15 \mathrm{Fi} / \mathrm{PF} 7685-7696 \\
3253 \mathrm{~W} 1-2\end{array}$ & Registres d'écrou, photographies. & 1940-1949 \\
\hline Charenton-le-Pont & Foyer d'orientation éducative (FOE) & 1957W 1-4 & $\begin{array}{l}\text { Fonctionnement : rapports annuels, cahiers et journaux de } \\
\text { bord. }\end{array}$ & 1971-1989 \\
\hline Chevilly-Larue & $\begin{array}{l}\text { Centre d'observation et de } \\
\text { rééducation (COR) }\end{array}$ & 4163W 1-1511 & $\begin{array}{l}\text { Fonctionnement: organisation générale, rapports } \\
\text { annuels, comptabilité. Pensionnaires : registres d'entrées } \\
\text { et de présence, fichiers, dossiers individuels. }\end{array}$ & 1807-2015 \\
\hline $\begin{array}{l}\text { Fontenay-sous- } \\
\text { Bois }\end{array}$ & $\begin{array}{l}\text { Unité éducative d'hébergement } \\
\text { diversifié (UEHD) }\end{array}$ & 4056W 1-56 & Prise en charge des mineurs : dossiers individuels. & 2004-2011 \\
\hline Fresnes & $\begin{array}{l}\text { Ecole de préservation, } \\
\text { Maison d'éducation surveillée } \\
\text { (MES), puis centre spécial } \\
\text { d'observation et d'éducation } \\
\text { surveillée (CSOES) }\end{array}$ & $\begin{array}{l}2 \mathrm{YY} 5,1836 \mathrm{~W}, \\
2656 \mathrm{~W}, 2742 \mathrm{~W} \\
3507 \mathrm{~W}\end{array}$ & $\begin{array}{l}\text { Ecole de préservation et MES : registres d'écrous, registres } \\
\text { de mouvements journaliers. } \\
\text { CSOES, fonctionnement : notes de services, statistiques; } \\
\text { suivi des mineurs : registres d'écrous, dossiers individuels. }\end{array}$ & 1904-1979 \\
\hline Nogent-sur-Marne & $\begin{array}{l}\text { Centre d'orientation éducative } \\
\text { (COE) }\end{array}$ & $\begin{array}{c}2032 W 1-201 \\
2547 W 1-35\end{array}$ & $\begin{array}{l}\text { Consultations: registre d'entrée, dossiers individuels. } \\
\text { Psychologie de l'enfant, étude : rapports. }\end{array}$ & 1961-1990 \\
\hline Villejuif & Centre d'éducation surveillée (CES) & $\begin{array}{c}\text { 1Mi 1711, } \\
\text { 15Fi/PF 7661-7684, } \\
\text { 3254W } 1\end{array}$ & Registres d'écrou, album photographique. & 1943-1949 \\
\hline Vitry-sur-Seine & $\begin{array}{l}\text { Centre d'orientation éducative } \\
\text { (COE) }\end{array}$ & 1950W 1-109 & Consultations : dossiers individuels, journaux de bord. & 1970-1979 \\
\hline
\end{tabular}

11 Les archives les plus anciennes proviennent de l'établissement pénitentiaire de Fresnes, qui accueille des filles depuis 1904 et des garçons depuis 1930. Structures dénommées respectivement école de préservation (depuis 1927) et maison d'éducation surveillée (MES), elles témoignent de la prise en charge des mineurs de région parisienne avant 1945, et notamment des premiers essais de triage menés dès 1930 à la MES.

Chronologiquement, suivent les centres de triage et d'observation créés au cours de la Seconde Guerre mondiale. Outre la MES de Fresnes, deux centres sont implantés dans ce qui deviendra le Val-de-Marne : à Charenton-le-Pont et Villejuif. Les archives de ces centres sont réduites aux registres d'écrou, et doivent être rapprochées de celles conservées par les archives de Paris en provenance des centres similaires des Tourelles, de la rue de Crimée et de la rue de Madrid.

Concernant la prise en charge des mineurs après la Seconde Guerre mondiale, le fonds du centre d'orientation et d'action éducative (COAE) d'Arcueil est à distinguer. Cette structure mêlant orientation et hébergement d'urgence est la seconde créée en France en 1959, après celle de Lille. Ses archives se démarquent par leur importance matérielle (212 articles), la longueur de la période couverte (1958-1992) et la diversité de leur contenu : en plus de la collection complète des dossiers de consultation d'orientation, sont conservés une vingtaine d'articles sur l'organisation et le fonctionnement du centre. Le fonds du COAE peut être comparé et complété par ceux des centres de 
Nogent-sur-Marne, Vitry-sur-Seine, ou du foyer d'action éducative de Charenton-lePont qui couvrent pour partie les mêmes périodes.

Enfin, pour les périodes plus récentes, les archives départementales sont encore peu riches puisque les dossiers sont toujours conservés dans les services qui les ont produits. Mais la collecte des fonds des établissements publics relevant de la PJJ est toujours d'actualité! Ainsi, en 2016, l'unité éducative d'hébergement diversifié de Fontenay-sous-Bois (UEHD) a effectué son premier versement constitué de dossiers individuels des années 2000.

15 Concernant la consultation, ces fonds sont soumis à des délais de communicabilité divers, qui peuvent aller de 50 ans (protection de la vie privée) à 120 ans (secret médical), en passant par celui de 100 ans (procédure judiciaire concernant des mineurs). Une demande de dérogation auprès des archives départementales est donc nécessaire pour effectuer une recherche historique.

Ces documents posent aussi la question de l'accès à son propre dossier et de l'accompagnement nécessaire à sa consultation. Ce point avait déjà été soulevé aux archives départementales du Val-de-Marne pour les dossiers individuels de l'Aide sociale à l'enfance (ASE) pour lesquels une procédure a été mise en place : le choix est de privilégier une consultation accompagnée par des professionnels, et ce sont donc les agents de l'ASE qui accueillent systématiquement l'usager.

Avant l'arrivée des archives du COR de Chevilly-Larue, la question de la consultation des dossiers des établissements relevant de la PJJ par le mineur concerné n'avait jamais été soulevée. Ce versement de dossiers récents et l'arrivée conjointe de demandes d'usagers a donné l'occasion de réfléchir à une procédure en s'inspirant de celle mise en place pour les dossiers de l'ASE: ainsi, même si l'accueil se fait aux archives départementales, la consultation se fait dans un bureau séparé de la salle de lecture, accompagné d'une éducatrice de la Protection judiciaire de la jeunesse qui a accepté de se déplacer afin d'expliquer au mieux les documents et de les replacer dans leur contexte. Ce mode d'accès n'a été testé qu'une fois et a convenu tant à l'usager qu'à la PJJ et aux archives départementales.

18 Au final, les fonds des structures relevant de la Protection judiciaire de la jeunesse aux archives du Val-de-Marne présentent une grande variété de modes de prise en charge des mineurs au $\mathrm{xx}^{\mathrm{e}}$ siècle en région parisienne, même si leur exploitation ne peut s'imaginer qu'en les mettant en lien avec les autres structures d'île-de-France. Il ne fait aucun doute qu'ils sont loin d'avoir révélé toute leur richesse et que les délais de communicabilité ne doivent pas être un frein aux diverses exploitations historiques qui pourront être imaginées! 


\section{AUTEURS}

\section{AGNÈS SEGUIN}

Agnès Seguin est archiviste, chargée de projet classement - description à la direction des archives départementales du Val de Marne.

\section{ZOÏ KYRITSOPOULOS}

Zoï Kyritsopoulos est cheffe du service des publics aux archives départementales du Val de Marne. 